\title{
Computation of the Spectral Density of Current Fluctuations in Bulk Silicon Based on the Solution of the Boltzmann Transport Equation
}

\author{
ALFREDO J. PIAZZA* and CAN E. KORMAN \\ Department of Electrical Engineering and Computer Science, The George Washington University, Washington, DC 20052
}

\begin{abstract}
Numerical simulation results for the spectral density of noise due to current fluctuations are presented. The mathematical framework is based on the interpretation of the equations describing electron transport in the semiclassical transport model as stochastic differential equations (SDE). Within this framework, it was previously shown that the autocovariance function of current fluctuations can be obtained from the transient solution of the Boltzmann transport equation (BTE) with special initial conditions. The key aspect which differentiates this approach from other noise models is that this approach directly connects noise characteristics with the physics of scattering in the semiclassical transport model and makes no additional assumptions regarding the nature of noise. The solution of the BTE is based on the Legendre polynomial method. A numerical algorithm is presented for the solution of the transient BTE. Numerical results are in good agreement with Monte Carlo noise simulations for the spectral density of current fluctuations in bulk silicon.
\end{abstract}

Keywords: stochastic differential equations, Boltzmann transport equation, noise, Legendre polynomials

\section{INTRODUCTION}

Noise is generally characterized by the spectral density of current or voltage fluctuations. In this paper, we present numerical simulation results for the autocovariance function and spectral density of current fluctuations based on a new noise model [1]. Employing the machinery of SDE, this model shows that key computations for the noise autocovariance function are reduced to the solution of the BTE with special initial conditions. Based on this novel approach, we study the influence of temperature and electric field on noise spectral density in bulk silicon. We also compare our results with those obtained using the
Monte Carlo technique [2]. The paper is organized as follows. Section II summarizes the noise model employed for the calculations. Section III describes the algorithm for the solution the transient BTE based on the Legendre polynomial method [3] and presents numerical results. The last section is devoted to the conclusions.

\section{NOISE MODEL BASED ON SDE}

According to semiclassical transport theory, an electron in a semiconductor drifts under the influence of electric field and experiences occasional random

\footnotetext{
* Corresponding author.
} 
changes in its momentum due to the scattering mechanisms in the crystal. This process is described in terms of the following SDE :

$$
\begin{aligned}
\frac{d \vec{x}}{d t}=\vec{v}(\vec{k})=\frac{1}{\hbar} \nabla_{\vec{k}} \varepsilon(\vec{k}), \frac{d \vec{p}}{d t} & =\hbar \frac{d \vec{k}}{d t}=-q \vec{E}+\vec{F}_{r}, \\
\text { and } \vec{F}_{r} & =\sum_{i} \hbar \vec{u}_{i} \delta\left(t-t_{i}\right),
\end{aligned}
$$

where $\vec{x}, \vec{v}, \vec{p}$ and $\vec{k}$ are the electron position, drift velocity, momentum and wave vector, respectively, $\vec{E}$ is the electric field, $\varepsilon(\vec{k})$ is the energy-wave vector relationship in the given energy band and $F_{r}$ is the random impulse force on the electron due to scattering. The random force is characterized by the transition rate $W(\vec{k}, \vec{k})$. Accordingly, the probability of scattering is given by

$$
\begin{array}{r}
\operatorname{Pr}\left\{t_{i}-t_{i-1}>\tau\right\}=\exp \left\{-\int_{t_{i-1}}^{t_{i-1}+\tau} \lambda\left(\vec{k}\left(t^{\prime}\right)\right) d t^{\prime}\right\}, \\
\lambda(\vec{k})=\int W\left(\vec{k}, \vec{k}^{\prime}\right) d \vec{k}^{\prime}
\end{array}
$$

where $\lambda(\vec{k})$ is the scattering rate. Therefore, given the electron wave vector $\vec{k}, \lambda(\vec{k}) \Delta t$ is the probability that a jump in momentum will occur in a small time interval $\Delta t$. Assuming that a scattering event has occurred at some time $t_{i}$, the probability density function for the amplitude of the jump is given by,

$$
\chi_{\vec{k}_{i}}\left(\vec{u}_{i}\right)=\frac{W\left(\vec{k}_{i}, \vec{k}_{i}+\vec{u}_{i}\right)}{\lambda\left(\vec{k}_{i}\right)}
$$

where $\vec{k}\left(t_{i}^{-}\right)=\vec{k}_{i}$ and $\vec{k}\left(t_{i}^{+}\right)=\vec{k}_{i}+\vec{u}_{i}$. Note that these are the same equations which are used to simulate the electron motion in Monte Carlo simulations.

The stochastic differential equations (1) together with Eqs. (2) and (3) define a compound Poisson process which is discontinuous in $\vec{k}$-space and is a Markov process. In stochastic differential equation theory, such a process is usually characterized by a transition probability density function, which satisfies the Kolmogorov-Feller forward equation. Employing the machinery of SDE theory it was previously shown that this function satisfies the BTE (see [4]).
Semiconductor noise is usually characterized by the spectral density of current fluctuations. The spectral density of current fluctuations is by definition the time Fourier transform of its autocovariance function. Since the instantaneous electron current is proportional to the electron momentum, the autocovariance function of current fluctuations can be computed directly from the autocovariance function of the electron momentum. In order to compute the autocovariance function of a stochastic process $k_{t}$, one needs to know the joint probability density function of the stochastic process at times $t+\tau$ and $t$ for all $\tau$. Since the stochastic process is stationary, the reference time $t$ is arbitrary and can be chosen to be zero for convenience. Consequently, it is sufficient to compute the joint probability density function $\rho\left(k_{\tau}, k_{0}\right)$. Employing conditional probabilities, the joint probability density function can be expressed as follows: $\rho\left(k_{\tau}, k\right)$ $=\rho\left(k_{\tau} \mid k\right) \rho_{0}(k)$. This conditional probability density function is nothing more than the transitional probability density function which characterizes the Markov process and it satisfies the KolmogorovFeller forward equation with the initial condition, $\left.k_{t}\right|_{\tau=0}=k$. On the other hand, the probability density function $\rho_{0}(k)$ satisfies the stationary Kolmogorov-Feller equation since the process is stationary and the initial reference time is arbitrary. Since the Kolmogorov-Feller equation is identical to the BTE, we conclude that the key computations for the noise spectral density are reduced to special initial value problems for the BTE.

The computation of the noise spectral density is based on this formalism and is described in [1]. According to this formalism, it is shown that the autocovariance matrix $\hat{K}_{k}^{*}(\tau)$ of the electron wave-vector can be computed as follows. For bulk noise computations, the space-independent BTE,

$$
\frac{\partial \vec{a}}{\partial \tau}(\vec{k}, \tau)-\frac{q}{\hbar} \vec{E} \cdot \nabla_{\vec{k}} \vec{a}=\int \vec{a}\left(\vec{k}^{\prime \prime}, \tau\right) W\left(\vec{k}^{\prime \prime}, \vec{k}\right) d \vec{k}^{\prime \prime}-\lambda(\vec{k}) \vec{a},
$$


is solved subject to the initial condition: $\left.a(\vec{k}, \tau)\right|_{\tau=0}=\vec{k} f_{\infty}(\vec{k})$, where $f_{\infty}(\vec{k})$ denotes the stationary solution. Here, equation (4) should be understood in a component-wise sense. The above solution is substituted into the following equation in order to evaluate the autocovariance matrix:

$$
\hat{K}_{\vec{k}}(\tau)=\int[\vec{k}]\left[\vec{a}(\vec{k}, \tau)-\langle\vec{k}\rangle f_{\infty}(\vec{k})\right]^{T} d \vec{k}, \tau \geq 0 .
$$

Since, the autocovariance function is a symmetric function of $\tau$, the solution of Eq. for $\tau \geq 0$ is sufficient.

\section{SOLUTION OF THE BTE EMPLOYING LEGENDRE POLYNOMIALS}

It is assumed that a single band distribution function accurately represents the state of the momentum space. The Herring-Vogt transformation is employed to map the coordinate system $\vec{k}$ into $\vec{k}^{*}$ which results in spherical equal energy surfaces: $\gamma(\varepsilon)=\varepsilon+\beta \varepsilon^{2}$ $=\hbar^{2} k^{* 2} / 2 m_{o}$. In $\vec{k}^{*}$ momentum space the direction of the average electron wave vector $\left\langle\vec{k}^{*}\right\rangle$ defines a symmetry axis $(\hat{z})$ and the density function dependence on momentum can be expressed in terms of only two independent variables: $\varepsilon$ and $\theta$. We expand $f\left(k^{*}, t\right)$ in Legendre polynomials according to:

$$
\begin{aligned}
f\left(\vec{k}^{*}, t\right)=f_{0}(\varepsilon, t)+k^{*} g & (\varepsilon, t) \cos \theta \\
& +k^{*^{2}} h(\varepsilon, t)\left(3 \cos ^{2} \theta-1\right) .
\end{aligned}
$$

In terms of this representation for $f\left(\vec{k}^{*}, t\right)$, the BTE can be formulated as follows :

$$
\begin{aligned}
\left\{\left(\frac{\partial f_{0}}{\partial t}(\varepsilon, t)-\right.\right. & \left.k^{*^{2}} \frac{\partial h}{\partial t}(\varepsilon, t)\right)+\cos \theta\left(k^{*} \frac{\partial g}{\partial t}(\varepsilon, t)\right) \\
+ & \left.\cos ^{2} \theta\left(3 k^{*^{2}}, \frac{\partial f_{0}}{\partial t}(\varepsilon, t)\right)\right\} \\
- & \left\{\frac { q } { \hbar } E \left[g+\cos \theta\left(\frac{4 \gamma^{1 / 2} \sqrt{2 m_{0}}}{\hbar} h\right.\right.\right. \\
& \left.\left.\left.+\frac{\hbar \sqrt{2} \gamma^{1 / 2}}{\sqrt{m_{0}} \gamma^{\prime}} \frac{\partial f_{0}}{\partial \varepsilon}\right)+\cos ^{2} \theta \frac{2 \gamma}{\gamma} \frac{\partial g}{\partial \varepsilon}\right]\right\}
\end{aligned}
$$

$$
\begin{gathered}
=\left\{f_{0}(\varepsilon, t) K_{\text {Acoustic }} \gamma^{1 / 2}(\varepsilon) \gamma^{\prime}(\varepsilon)\right. \\
+\sum_{j} K_{\text {Optical }_{j}}\left(\left.f_{0}(\psi, t) \gamma^{1 / 2}(\psi) \gamma^{\prime}(\psi)\right|_{\psi=\varepsilon+\hbar \omega_{j}}\right. \\
\left.\left.+\left.e^{\hbar \omega_{j} / K T} f_{0}(\psi, t) \gamma^{1 / 2}(\psi) \gamma^{\prime}(\psi)\right|_{\psi=\varepsilon-\hbar \omega_{j}}\right)\right\} \\
\quad-\left\{f _ { 0 } ( \varepsilon , t ) \left[K_{\text {Acoustic }} \gamma^{1 / 2}(\varepsilon) \gamma^{\prime}(\varepsilon)\right.\right. \\
+\sum_{j} K_{\text {Optical }_{j}}\left(\left.\gamma^{1 / 2}(\psi) \gamma(\psi)\right|_{\psi=\varepsilon+\hbar \omega_{j}}\right. \\
\left.\left.\left.\quad+\left.e^{\hbar \omega_{j} / K T} \gamma^{1 / 2}(\psi) \gamma(\psi)\right|_{\psi=\varepsilon-\hbar \omega_{j}}\right)\right]\right\}
\end{gathered}
$$

Recalling that the Legendre polynomials are orthogonal, Eq. (7) can be solved for the corresponding coefficients independently. This results in a system of three coupled differential-difference equations for the functions $f, g$, and $h$, in terms of $t$ and $\varepsilon$. By defining $f\left(\vec{k}^{*}, t_{i}\right)=f_{i}$, and $f\left(\vec{k}^{*}, t_{i}-\Delta_{t}\right)=f_{i-1}$, the solution of the system starts with the following finite-difference in time approximation:

$$
\begin{aligned}
& \frac{\partial f_{0}}{\partial t}(\varepsilon, t) \approx \frac{f_{0_{i}}(\varepsilon)-f_{0_{i-1}}(\varepsilon)}{\Delta_{t}}, \\
& \frac{\partial g}{\partial t}(\varepsilon, t) \approx \frac{g_{i}(\varepsilon)-g_{i-1}(\varepsilon)}{\Delta_{t}}, \\
& \frac{\partial h}{\partial t}(\varepsilon, t) \approx \frac{h_{i}(\varepsilon)-h_{i-1}(\varepsilon)}{\Delta_{t}} .
\end{aligned}
$$

As a result, an expression of the form depicted in Eq. (9) is derived for $f_{o_{i}}$, in which $A$ and $B$ represent general linear operators.

$$
A\left(f_{0_{i}}, f_{0_{i}}\left(\varepsilon \pm \hbar \omega_{j}\right)\right)=B\left(f_{0_{i-1}}, g_{i-1}, h_{i-1}\right)
$$

At the $i^{\text {th }}$ iteration the right hand side of Eq. (9) is evaluated by substituting the solution of the previous $(i-1)^{\text {th }}$ iteration. The function $f_{o_{i}}(\varepsilon)$ is then computed by approximating the left hand side of this equation using the standard finite difference method. Then the functions $g_{i}(\varepsilon), \frac{\partial g_{i}}{\partial \varepsilon}, h_{i}(\varepsilon)$ and $\frac{\partial h_{i}}{\partial_{\varepsilon}}$ are obtained from $f_{0 i}(\varepsilon), f_{0 i-1}(\varepsilon), g_{i-1}(\varepsilon)$ and $h_{i-1}(\varepsilon)$.

Figs. (1a) and (1b) show computations for the longitudinal autocovariance function at two different temperatures. As expected, both plots show a narrowing in the correlation function as the electric field is 
$\mathrm{E}=4.17 \mathrm{kV} / \mathrm{cm}$ are repeated in each plot for comparison at different temperatures. Although the shapes of these curves are similar, the time scales are significantly different which shows the role of temperature as an important source of noise. Figure (1c) shows the spectral density corresponding to Fig 1c. These results are in very good agreement with published results computed using Monte Carlo technique [2].

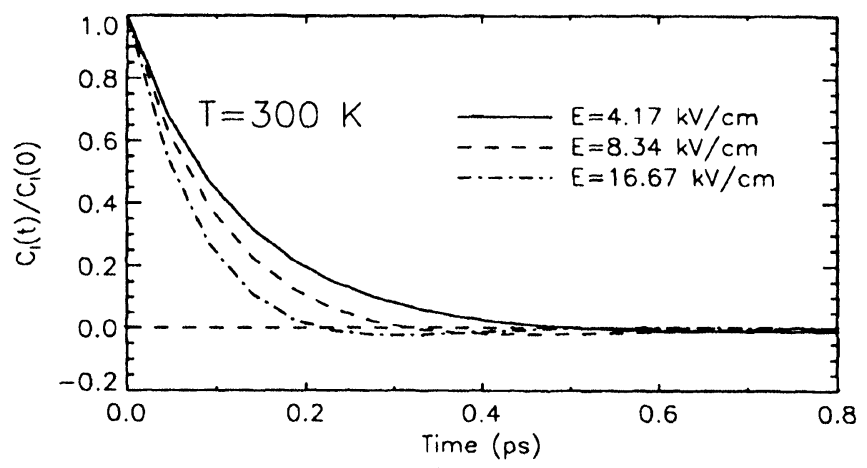

(a)

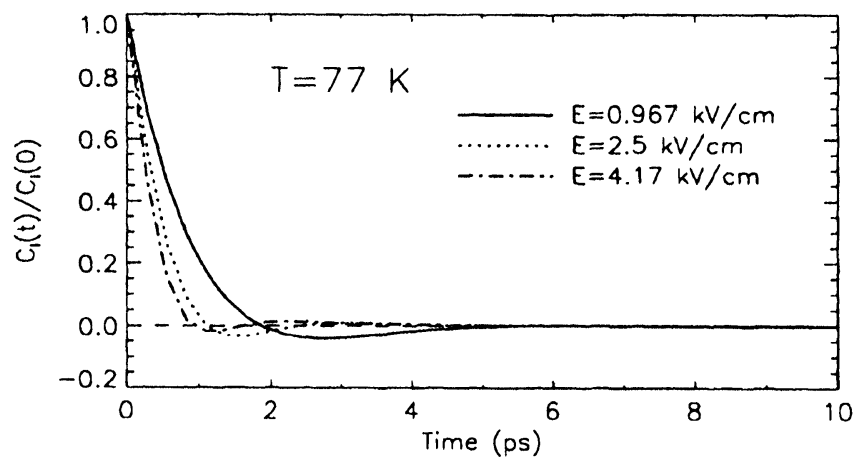

(b)

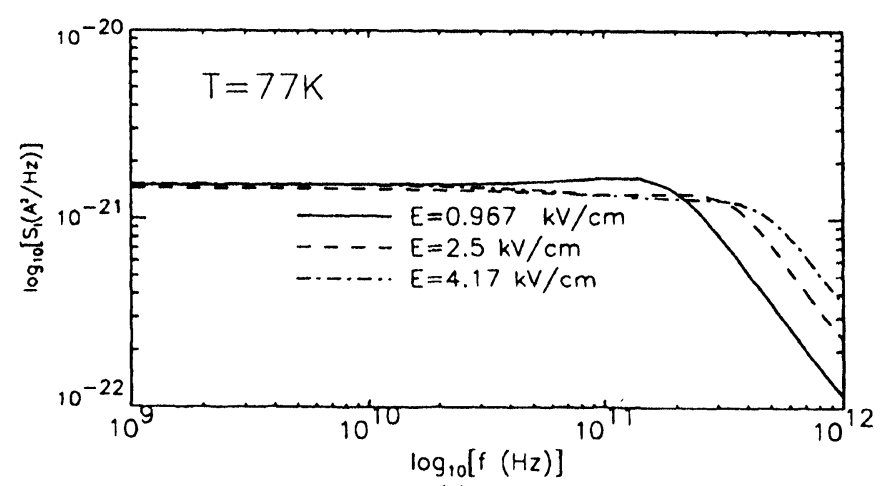

(c)

FIGURE 1 Figures (1a) and (1b) show calculations for the normalized longitudinal autocovariance function for two different temperatures. Figure (1c) shows the corresponding spectral density function to (1b) 


\section{CONCLUSION}

A new noise model was briefly reviewed. It was pointed out that the key computations for the autocovariance function are reduced to a special initial value problem for the transient BTE. An algorithm for the solution of the BTE based on the first three Legendre polynomials was described. Numerical results for the spectral density and autocovariance function of current fluctuations due to elastic acoustic and optical phonon scattering under static electric fields were presented for silicon. The results are in excellent agreement with results obtained using Monte Carlo techniques due to the fact that both approaches are strictly within the framework of the semiclassical transport theory. Hence, the noise model based on the theory of SDE is demonstrated to be a viable, accurate and computationally efficient alternative to the Monte Carlo technique for the computation of noise characteristics in semiconductors.

\section{References}

[1] C. E. Korman and I. D. Mayergoyz, "Semiconductor Noise in the Framework of Semiclassical Transport," in preparation.

[2] L. Varani, L. Reggiani, T. Kuhn, T. Gonzalez and D. Pardo, "Microscopic Simulation of Electronic Noise in Semiconductor Materials and Devices," IEEE Trans. on Electron Devices, Vol. 41, No. 11, pp. 1916-1926, November 1994.

[3] N. Goldsman, L. Henrickson and J. Frey, "A Physics-Based Analytical/Numerical Solution to the Boltzmann Transport Equation for Use in Device Simulation," Solid-St. Electron Vol. 34, No 4, pp. 389-396, 1991

[4] C. E. Korman and I. D. Mayergoyz, "Application of Stochastic Differential Equation Theory to Semiconductor Transport," Proceedings of the International Workshop on Computational Electronics, pp. 159-162, University of Illinois at Urbana-Champaign, May, 1992.

\section{Biographies}

Can E. Korman was born in Silver Spring, Maryland. He received the B.S., M.S. and Ph.D. degrees in Electrical Engineering from the University of Maryland in 1985, 1987 and 1990, respectively. In 1991, he joined the Department of Electrical Engineering and Computer Science at the George Washington University, Washington, DC where he is currently an Assistant Professor.

His current research is in the area of numerical simulation of semiconductor devices, semiconductor noise characterization, parallel algorithms, stochastic differential equations, VLSI and magnetic viscosity. $\mathrm{He}$ is the recipient of the National Science Foundation Research Initiation Award. He has also received the following teaching awards at the George Washington University: the Eta Kappa Nu Association Teacher of the Year Award for 1993-94 and the Engineer's Council Faculty of the Year Award for 1994-95. He is a member of the IEEE Electron Devices Society and the Circuits and Systems Society.

Alfredo Piazza was born in Lima, Peru, in 1963. $\mathrm{He}$ received the B.S. degree in industrial engineering from the Pontificia Universidad Catolica del Peru in 1988. In 1995 he obtained the M.S. degree in electrical engineering with concentration in electrophysics from the George Washington University. He continues at the George Washington University, working toward the Ph.D. in electrical engineering with concentration in systems science, networks and controls. His research concentrates in the numerical simulation of semiconductor devices. His current research is on the topic of noise characterization of semiconductor materials by means of a direct solution of the Bolzmann Transport Equation. 

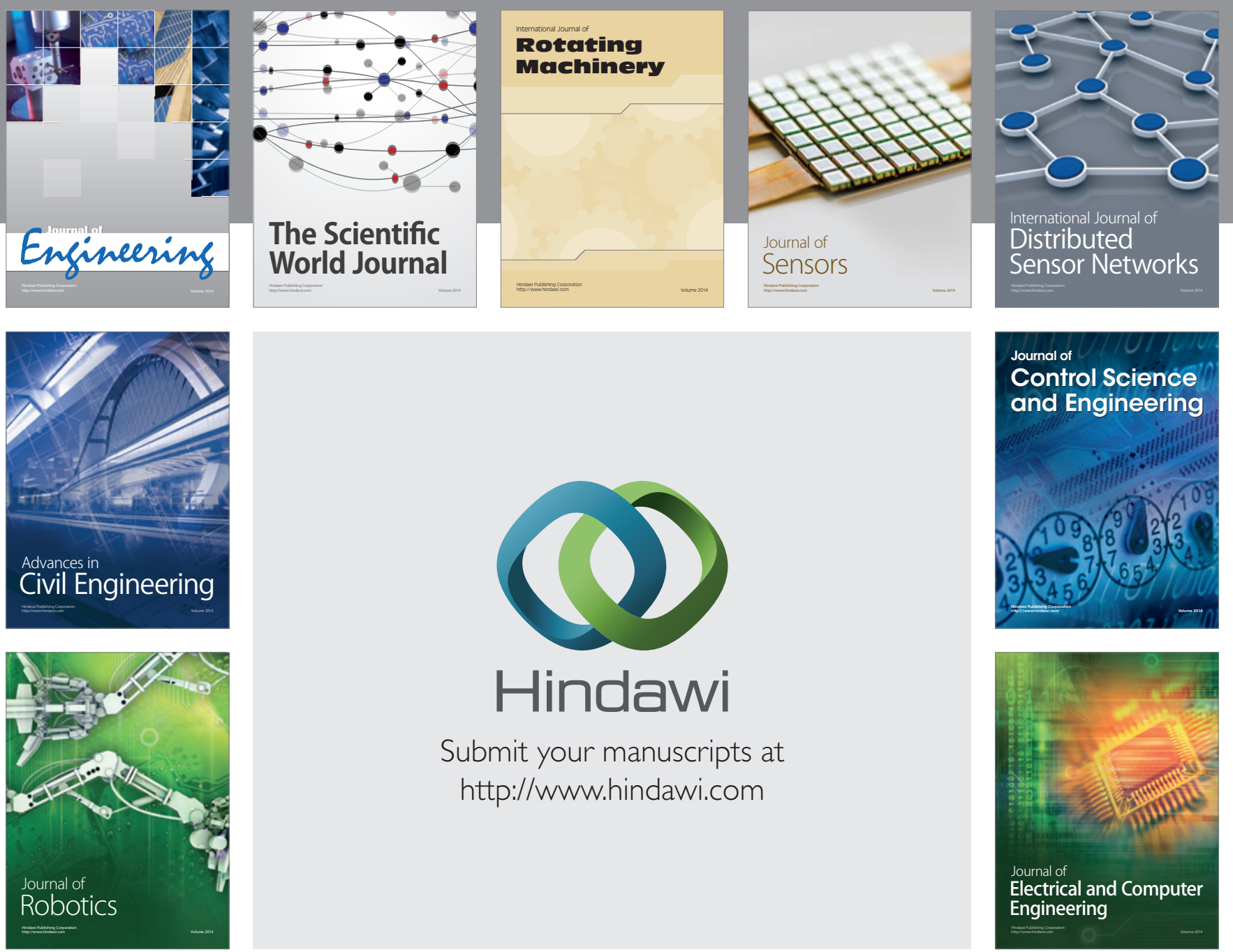

Submit your manuscripts at

http://www.hindawi.com
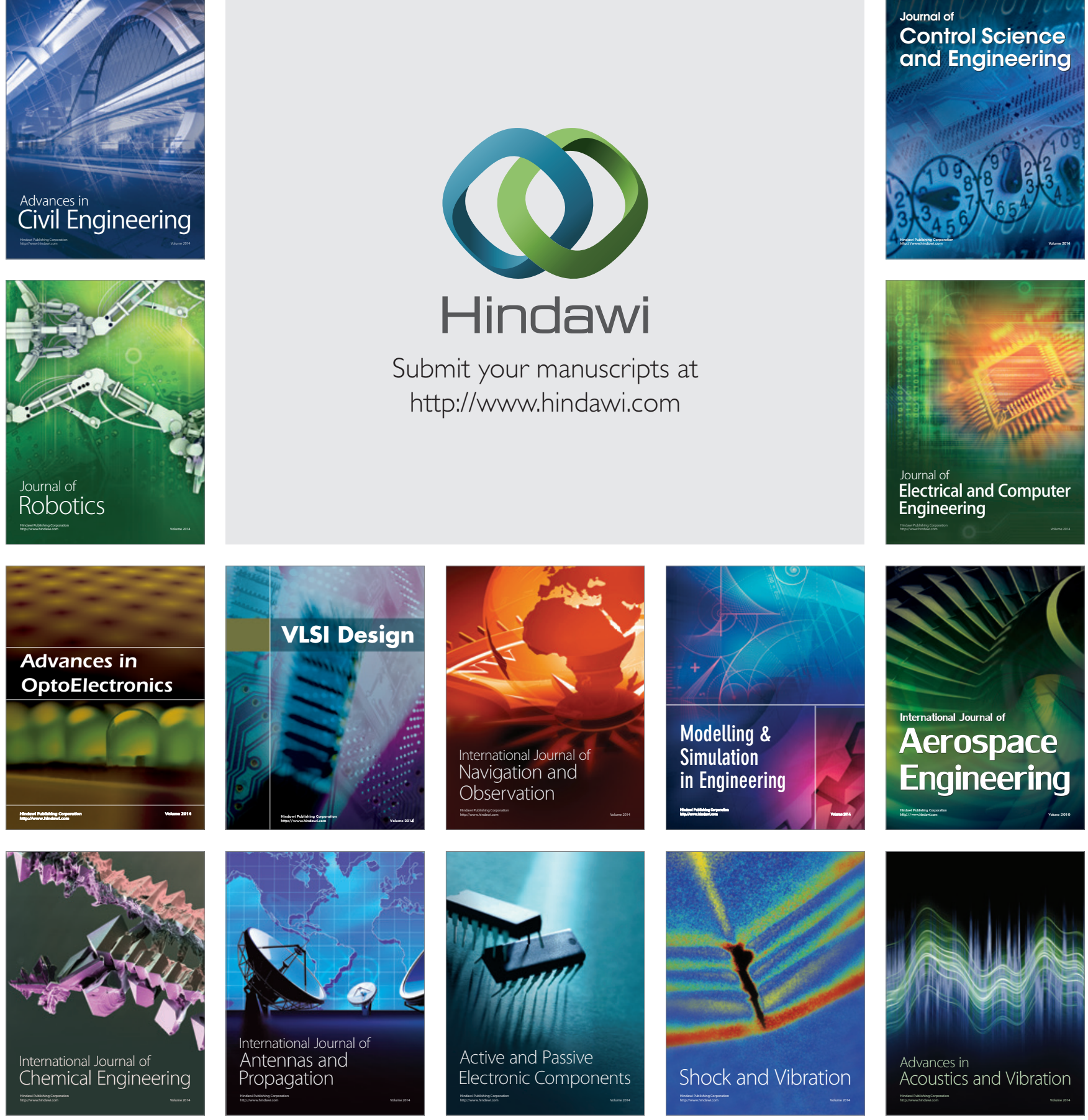